\title{
ESTUDIO DESCRIPTIVO Y EVALUACIÓN DE UNA MUESTRA DE TRANSEÚNTES MARGINADOS RESIDENTES EN UN CENTRO DE ACOGIDA
}

\author{
M. ÁNGELES JIMÉNEZ TALLÓN \\ Universidad de Murcia \\ (Recibido el 11 de diciembre de 1998)
}

\begin{abstract}
Se ha estudiado una muestra de 106 sujetos varones: 55 de ellos son transeúntes marginados residentes en un centro de acogida y el resto reside en su entorno socio-familiar habitual. Ambos grupos son equiparables en cuanto a edad, nivel educativo y origen social.

Se evaluaron los rasgos de personalidad y otras características psicosociales, mediante el 16PF, entrevista personal, test de recursos personales, test de alcoholismo y escala de autoestima. La comparación entre ambos grupos reveló la existencia de diferencias significativas en estrés psicosocial, recursos personales, inteligencia, estado civil, edad, afectotimia, dependencia y actividad laboral de la madre.
\end{abstract}

Palabras clave: Evaluación, transeúntes marginados, rasgos personalidad.

\section{Descriptive study and assessment of a sample of transient homeless men living in a refuge centre}

A sample of 106 men has been studied: 55 of them are transient homeless men men in a refuge centre and the rest live in their own usual family environment. Both groups are similar in age, educational level and social background.

Personality features and other psychosocial characteristics were evaluated by $16 \mathrm{PF}$, personal interview, test of personal resources, test of alcoholism and self-esteem scale. The comparison of both groups showed the existence of significant differences in psychosocial stress, personal resources, intelligence, marital status, age, illness of affection, dependence and mother's job.

Key words: Assessment, transient homeless, personality features.

\section{INTRODUCCIÓN}

El concepto de "transeúnte marginado" es de reciente definición aunque no sea un hecho social nuevo. El «transeuntismo" como fenómeno sociológico está directamente relacionado con la ausencia de recursos, aunque ha sido muy escasa la aportación teórica que aborda conceptualmente este hecho como una de las variantes de la marginación social. Según

Correspondencia: M. Ángeles Jiménez Tallón, Departamento de Personalidad, Evaluación y Tratamientos Psicológicos, Universidad de Murcia, Apdo. de Correos 4021, 30008 Murcia.

ajtallon@fcu.um.es. el estudio realizado por la concejalía de Servicios Sociales del Ayuntamiento de Madrid (1986), los transeúntes marginados son: «aquellos individuos aislados que se encuentran con mínimos medios económicos y en paro laboral, que no disponen de un habitat propio para alojarse, que han roto con los lazos familiares, recurriendo a las Instituciones sociales para sobrevivir y sin poseer recursos personales para afrontar su situación".

En estos individuos no existe conciencia de grupo y adquiere su máximo significado el concepto de aislamiento social. El transeúnte marginado está desprovisto de vínculos sociales que le res- 
palden en sus actuaciones y actitudes. Al estar desligado también de las relaciones familiares, carece del apoyo que supone el grupo consanguíneo y queda ajeno a la solidaridad familiar.

Por todo lo dicho se aprecia claramente un problema de adaptación en estos sujetos. La adaptación es un fenómeno por el cual el individuo se relaciona con el entorno que le rodea en sus múltiples aspectos: geográficos, biológicos, económicos y sociales. La adaptación de una persona a su entorno social supone que esa persona ha interiorizado suficientemente los modelos, símbolos y valores de su medio ambiente en su propia estructura personal, para comunicar de forma adecuada con los miembros de las colectividades de las que forma parte, de tal forma que se pueda afirmar que ese sujeto pertenece realmente a una determinada colectividad (García Roca et al., 1991).

No obstante, la acomodación de un ser humano a las exigencias grupales y sociales no puede perturbar de manera excesiva las necesidades del individuo, pues es imprescindible una cierta gratificación de las necesidades y deseos de las personas para que se pueda establecer una relación suficientemente armoniosa entre el sujeto y su entorno. Si este tipo de adaptación no tiene lugar surgen las situaciones de inadaptación y marginación social con respecto al entorno socio-ambiental (Caballo, 1987). Sin embargo, estas situaciones de inadaptación y marginación no tienen porqué ser irreversibles y pueden ser modificadas siempre que tenga lugar una intervención adecuada sobre los sujetos en conflicto psicosocial.

Como ha indicado Kelly (1992), estas intervenciones alcanzarán sus objetivos si se trabaja en tres apartados específicos: las habilidades sociales que faciliten la relación del sujeto consigo mismo y con los demás, el aprendizaje de unas técnicas de comunicación aceptable con el entorno y finalmente conseguir que el individuo que ha visto gravemente alterada la relación con su contexto socioambiental, logre percibir un sentido vital que mejore su autoconcepto y estimule sus posibles vivencias personales.

\section{Aspectos psicológicos en los transeúntes marginados}

La problemática psicológica suele ir ligada a una problemática social que ha incidido negativamente sobre el sujeto, que puede acabar padeciendo serias disfunciones mentales . Según el estudio del Ayuntamiento de Madrid (1986), el aspecto relacional en el transeunte marginado describe una situación de insociabilidad permanente: los amigos no existen y sólo se desarrollan contactos transitorios; el elemento que une a los transeúntes marginados, y que se convierte en centro de compañerismo, es el consumo de alcohol. El aspecto familiar se traduce en un desapego más o menos absoluto con respecto a los parientes más próximos y colaterales, y el desarraigo es el concepto que mejor define la relación familiar ; desarraigo que en este caso va unido a la ilocalización espacial característica del nomadismo urbano e interurbano, pero que también incide en otra serie de variables psicológicas y ambientales (Porot, 1954; Jiménez Tallón, 1987). La importancia del papel de los lazos socio-familiares ya fue señalada por Cassel (1974) y Cobb (1976) pues constituyen significativas redes de apoyo social que amortigüan los efectos adversos sobre el equilibrio emocional del individuo, por lo cual, la ruptura de estas redes incide de manera importante en la aparición de diferentes trastornos de tipo emocional.

Por nuestra parte, la aplicación de un «Programa de intervención psicosocial sobre transeúntes marginados» llevado a cabo en un Centro de acogida de Murcia 
(Madrigal; Jiménez Tallón; y Madrigal 1996), nos llevó a investigar la posible existencia de unos rasgos de personalidad predominantes en estos sujetos, y sus características psicosociales específicas, así como la presencia de Estrés psicosocial, que hubiesen podido influir en sus hábitos de transeuntismo y marginalidad, incidiendo asímismo en su entrada en el Centro de acogida. Nos planteamos, en consecuencia, analizar las posibles diferencias existentes entre el grupo de transeúntes marginados residentes en el Centro de acogida y otro de personas de nivel socio cultural medio/bajo que residiesen en su entorno habitual socio familiar, para lo cual nos propusimos:

a) Obtener información sobre los rasgos de personalidad y otras variables psicosociales en una muestra de transeúntes marginados residentes en el Centro de acogida.

b) Investigar las variables predictoras de la posibilidad de convertirse en transeúnte marginado y precisar ser residente en un Centro de acogida.

c) Estudiar las variables que diferencian significativamente el colectivo de transeúntes marginados residentes en el Centro, de otro de similares condiciones de edad y nivel social y de instrucción (medio/bajo), residente en su entorno sociofamiliar habitual y a los que se aplicase una evaluación idéntica.

\section{MATERIAL Y MÉTODO}

\section{Sujetos}

Se han estudiado 106 sujetos varones, comprendidos entre los 25 y 60 años, todos los cuales carecen de instrucción media y superior al igual que sus familias de origen. De los 106 sujetos estudiados, 55 residían en el Centro de acogida por carecer de recursos económicos y son considerados como transeúntes marginados y los 51 restantes residen en su entorno sociofamiliar habitual, habiendo sido seleccionados de forma aleatoria entre sujetos varones que reunían las características citadas de carecer de instrucción media y superior al igual que sus familias de orígen y residentes todos ellos en zonas urbanas de las provincias de Murcia y Alicante. No se incluyeron mujeres en el estudio porque su presencia en el Centro de acogida se reducía a 5 señoras mayores de 65 años.

\section{Instrumentos de evaluación}

Todos los sujetos de la muestra cumplimentaron los siguientes instrumentos de forma individualizada:

1. Entrevista personal abierta para conocer la Historia sociofamiliar y el análisis psicobiográfico del sujeto evaluado. Se trata de una entrevista diseñada para la población de residentes y utilizada de forma habitual en el centro.

2. Cuestionario 16 PF de Cattell en su forma C para sujetos con formación media-baja. Como es sabido este cuestionario detecta 16 rasgos primarios básicos y 4 factores de segundo orden. Los primeros son los siguientes: Sizotimia-Afectotimia, B. Inteligencia, C. Fuerza del yo. E. Sumisión-Dominancia, F. DesurgenciaSurgencia (sobriedad-Entusiasmo), G. Fuerza del superyo, H. Trectia-Parmia (cohibido Atrevido), I. Harria-Premsia (Sensibilidad dura-blanda), L. Alaxia-Protensión (Confiado-Suspicaz), M. Praxernia-Autia (Práctico-Bohemio), N. (SencillezAstucia), O. Tendencia a la culpabilidad, Q1. Conservadurismo-Radicalismo, Q2. Adhesión al grupo-Autosuficiencia, Q3. Baja integración-Control autoimagen, Q4. Poca-Mucha tensión érgica. En cuanto a los factores de segundo orden, son: QI. Ansiedad baja-alta. QII. Introversión-extra- 
versión. QIII. Poca-mucha socialización controlada. QIV. Dependencia-Independencia.

3. Para la evaluación de la autoestima se aplicó una forma abreviada de la Escala de Rosenberg (Rosenberg 1979), que mediante 10 ítems recoge la percepción que tiene el sujeto de sí mismo.

4. Test de implicación en el alcohol (Mother y Weitz 1989), que valora el nivel de alcoholismo del sujeto.

5. Test de recursos personales (Mother y Weitz 1989) para conocer los apoyos familiares, sociales, afectivos y grupales así como habilidades que poseían los sujetos estudiados.

6. Evaluación del sujeto según el DSM-IV :Evaluación de la actividad global (EEAG), Escala de intensidad del estrés psicosocial, alteraciones somáticas $\mathrm{y}$ trastornos mentales.

\section{Procedimiento}

A todos los sujetos de la muestra se les aplicaron las pruebas mencionadas anteriormente, mediante entrevista personal y realizadas por psicólogos. A los residentes en el Centro de acogida se les aplicó por las mañanas en su horario de talleres ocupacionales y a los no residentes en las horas que tenían libres en sus actividades.

La profesión del sujeto y de sus padres se valora en una escala de 0 a 4 . El nivel de estudios se valora en una escala de 0 a 2, incluyendo como límite Bup y FP aunque estén sin concluir. El nivel de autoestima, el alcoholismo y los recursos personales se valoran según la puntuación obtenida en los cuestionarios correspondientes.

\section{Análisis de datos}

Para la comparación de los sujetos en las variables sociodemográficas, psicopa- tológicas y psicosociales se utilizaron pruebas paramétricas ( $t$ de Student) y no paramétricas (chi cuadrado), según la naturaleza de las variables analizadas.

Para obtener las variables predictoras del hecho de ser posible residente en un Centro de acogida, se aplicó un Análisis de Regresión líneal múltiple del módulo MGLH de SYSTAT, tomando como variable dependiente "Internamiento». A continuación se realizó un Análisis discriminante por pasos del paquete estadístico BMDP con el objeto de obtener las variables que discriminasen de forma significativa a los dos grupos estudiados de residentes y no residentes en un Centro de acogida.

\section{RESULTADOS}

El análisis de las variables sociodemográficas mostró diferencias significativas entre ambos grupos de sujetos residentes y no residentes en el Centro de acogida. Respecto al estado civil aparecen diferencias significativas en los datos referentes a sujetos casados, claramente superiores en el grupo de los no residentes. Por el contrario los separados/viudos y solteros son significativamente más numerosos en el grupo de los residentes. En la variable referente a cualificacion laboral aparece un porcentaje doble de sujetos integrados en operarios y servicios en el grupo de residentes que en el de no residentes y por el contrario son significativamente más numerosos los empleados, funcionarios y comerciantes en el grupo de los no residentes. En cuanto al nivel de estudios, los que carecen de ellos predominan en el grupo de los residentes y los que poseen formación de estudios primarios y secundarios son superiores en el colectivo de los no residentes.

El análisis de las variables psicopatológicas muestra valores significativa- 
mente superiores en alcoholismo en el grupo de los transeúntes marginales que en los sujetos que viven en su entorno familiar. También el nivel de trastornos mentales fue significativamente mayor en el colectivo de los transeúntes que en el de los sujetos del grupo control. Sin embargo no hubo diferencias significativas en cuanto a la presencia de trastornos somáticos entre ambos grupos. La Tabla 1 recoge las características sociodemográficas y psicopatológicas de los sujetos analizados

En cuanto a las características psicosociales evaluadas, los resultados obtenidos muestran diferencias significativas entre ambos grupos en el sentido siguiente: El grupo de no residentes presentó valores más elevados en recursos personales, autoestima y evaluación de la actividad global que el grupo de residentes, mientras que estos presentan un mayor nivel de estrés psicosocial. Estos resultados se resumen en la Tabla 2.

Como variables predictoras de internamiento, el Análisis de Regresión líneal múltiple incluyó las siguientes: Estrés, Recursos personales, Estado civil, Edad, Inteligencia (B), Sizotimia (A), Dependencia (Q2) y Profesión de la madre. Los coeficientes de cada una de estas variables se exponen en la Tabla 3.

Tabla 1. Características sociodemográficas y psicopatológicas

\begin{tabular}{|c|c|c|c|}
\hline & \multirow{2}{*}{$\begin{array}{c}\text { Residentes Centro Acogida } \\
N=55\end{array}$} & \multicolumn{2}{|c|}{ No residentes } \\
\hline & & $N=51$ & $p<$ \\
\hline \multicolumn{4}{|l|}{ Estado Civil } \\
\hline $\begin{array}{l}\text { Casados } \\
\text { Separados/Viudos } \\
\text { Solteros }\end{array}$ & $\begin{array}{r}0 \% \\
45,5 \% \\
54,5 \%\end{array}$ & $\begin{array}{r}66,7 \% \\
7,8 \% \\
25,5 \%\end{array}$ & $\begin{array}{l}0,01 \\
0,01 \\
0,01\end{array}$ \\
\hline \multicolumn{4}{|l|}{ Nivel Estudios } \\
\hline $\begin{array}{l}\text { Sin estudios } \\
\text { Est. Primarios } \\
\text { Est. Secundarios }\end{array}$ & $\begin{array}{l}12,7 \% \\
58,1 \% \\
29 \%\end{array}$ & $\begin{array}{r}0 \% \\
68,6 \% \\
31,4 \% \\
\end{array}$ & $\begin{array}{l}0,05 \\
0,05 \\
\mathbf{0 , 0 5} \\
\end{array}$ \\
\hline \multicolumn{4}{|l|}{ Cualificación Laboral } \\
\hline $\begin{array}{l}\text { Obreros/Servicios } \\
\text { Empleados } \\
\text { Funcionarios } \\
\text { Comerciantes } \\
\end{array}$ & $\begin{array}{rr}89 & \% \\
9 & \% \\
0 & \% \\
1,8 & \% \\
\end{array}$ & $\begin{array}{r}43,1 \% \\
21,6 \% \\
3,9 \% \\
33 \% \\
\end{array}$ & $\begin{array}{l}0,01 \\
0,01 \\
0,01 \\
0,01 \\
\end{array}$ \\
\hline \multicolumn{4}{|c|}{ Características Psicopatológicas } \\
\hline $\begin{array}{l}\text { Atcoholismo } \\
\text { Trastornos mentales } \\
\text { Trastornos somáticos }\end{array}$ & $\begin{array}{c}65,5 \% \\
49 \% \\
5,5 \%\end{array}$ & $\begin{array}{l}15,7 \% \\
11,8 \% \\
31,4 \%\end{array}$ & $\begin{array}{l}0,01 \\
0,01 \\
-\end{array}$ \\
\hline
\end{tabular}

Tabla 2. Características Psicosociales

\begin{tabular}{|c|c|c|c|c|c|c|c|}
\hline & \multicolumn{3}{|c|}{$\begin{array}{c}\text { Residentes Centro } \\
\quad(N=55)\end{array}$} & \multicolumn{4}{|c|}{$\begin{array}{c}\text { No Residentes } \\
(N=51)\end{array}$} \\
\hline & $\%$ & Media & D.T. & $\%$ & Media & D.T. & $p<$ \\
\hline Recursos Personales & 1,8 & 2,13 & 1,22 & 92,2 & 6,63 & 1,37 & 0,01 \\
\hline Autoestima & 41,8 & 11,73 & 3,65 & 94,1 & 17,04 & 2,87 & 0,01 \\
\hline Estrés & 94,5 & 4,33 & 0,58 & 13,7 & 1,59 & 0,92 & 0,01 \\
\hline EEAG & 29 & 61,27 & 11,44 & 94,1 & 81,18 & 8,11 & 0,01 \\
\hline
\end{tabular}


Tabla 3. Variables predictoras de internamiento en un Centro de Acogida

\begin{tabular}{lccc}
\hline \multicolumn{1}{c}{ Variable } & Coeficiente Estandarizado & Coeficiente & $p<$ \\
\hline Estrés & $-0,135$ & $-0,421$ & 0,001 \\
Recursos Personales & 0,056 & 0,292 & 0,001 \\
Estado Civil & 0,114 & 0,194 & 0,001 \\
Edad & $-0,006$ & $-0,127$ & 0,008 \\
B (Inteligencia) & 0,037 & 0,125 & 0,009 \\
A (Sizotimia) & 0,030 & 0,111 & 0,007 \\
Q2 (Dependencia) & 0,019 & 0,084 & 0,033 \\
Profesión Madre & 0,031 & 0,065 & 0,078 \\
Constante & 1,374 & 0,000 & 0,000 \\
\hline
\end{tabular}

Según estos resultados, los sujetos con mayor estrés psicosocial, menos recursos personales, sin pareja, con mayor edad, menor inteligencia, menor capacidad afectiva, mayor dependencia de la aprobación del grupo y menor nivel de actividad laboral por parte de la madre, son los que presentan mayor probabilidad de concluir siendo transeúntes marginales y precisar internamiento en un Centro de acogida.

A continuación se realizó un análisis discriminante, paso a paso, tomando como variable dependiente el ser o no residente en el Centro de acogida, obteniéndose como resultado que las variables que más discriminaban el grupo de transeúntes marginados del de residentes en su entorno familiar fueron por este orden: Estrés, Recursos Personales, Inteligencia (B), Estado civil, Edad, Sizotimia-Afectotimia (A), Dependencia-Autosuficiencia (Q2) y Profesión de la madre.

Según las matrices de clasificación, el $100 \%$ de Transeúntes-marginados fue incluido en su grupo de orígen. En el caso del grupo de no residentes, el $98 \%$ fue incluído correctamente en su grupo de referencia. La tasa total de clasificación fue del $99,1 \%$, lo que indica que los valores de las 8 variables introducidas diferencian claramente entre ambos grupos.

Según estos resultados, el grupo de Transeúntes marginados, está formado por sujetos con alto nivel de estrés psicosocial, con escasos recursos personales, inteligencia baja, sin pareja estable, con edad superior al grupo de no residentes, características más significativas en aislamiento y reserva así como en dependencia de la aprobación del grupo y por último es significativamente menor en el grupo de residentes el número de madres que trabajan fuera de casa.

\section{DISCUSIÓN}

Nuestros resultados han arrojado un perfil del Transeúnte-marginado que reside en un Centro de acogida que presenta las siguientes características: Carece de pareja estable, presenta un nivel de estudios inferior al del grupo control, cualificación profesional baja, alcoholismo, trastornos mentales, carece de recursos personales (redes de apoyo social familiar, grupal o de habilidades sociales), posee baja autoestima, obtiene baja puntuación en la Escala de Evaluación de la actividad global en cuanto a actividad psicológica, social y laboral (EEAG) y presenta un alto nivel de estrés psicosocial. Sus características personales muestran un mayor aislamiento y reserva, son menos afectuosos, más dependientes de la aprobación del grupo y han obtenido menor puntuación en inteligencia que los sujetos no residentes.

Estos resultados son en parte coincidentes con los obtenidos por Díez Tomé y Vielva (1990) en un estudio sobre transeúntes marginados en Cantabria en el 
que exponen una serie de factores precipitantes del transeuntismo y que son indicadores de ruptura familiar, laboral y comunitaria . Entre otros, estar soltero, viudo o separado, ser hijo de familia desestructurada o numerosa, llevar más de un año en el paro, realizar actividades estacionales o marginales, carecer de amigos, haber estado internado en instituciones, utilizar centros para marginados durante largos periodos y residir en núcleos urbanos. Esta ruptura se puede presentar gradualmente hasta llegar a la desorientación espacial total y a la práctica del nomadismo urbano e interurbano. En esta situación, la Institución de acogida supone el seguro de mantenimiento vital y se identifica como el espacio de referencia más importante para estos individuos, al constituirse en el lugar de seguridad ante las inclemencias exteriores.

En el estudio sobre Transeúntes marginados de Vielva (1989), se consigna el malestar psicológico existente en el colectivo de transeúntes marginados analizado, debido al alto nivel de ansiedad que presentan y al fuerte nivel de estrés experimentado (González de Rivera 1979), pues los transeúntes marginados manifiestan mayor número de acontecimientos estresantes y comienzan a experimentarlos desde más jóvenes y de forma más continuada que la población normal (Jiménez Tallón, 1988). Todo esto va unido a un elevado número de tentativas de suicidio, a alcoholismo, drogadicción y un autoconcepto altamente negativo. La comparación entre grupos de población general y transeúntes marginados (Vielva 1992), pone de manifiesto también diferencias significativas en la aparición de estados de ánimo depresivos y alteraciones emocionales, más acentuadas en los transeúntes marginados.

Uno de los datos obtenidos en este trabajo que resulta llamativo es la relación entre el hecho de ser transeúnte margi- nado residente en un Centro de acogida y la actividad laboral de sus madres, apreciándose diferencias significativas en este aspecto entre los grupos de residentes en el Centro de acogida y los residentes en su entorno sociofamiliar habitual, presentando estos últimos un mayor porcentaje de madres con una actividad profesional.

En definitiva, la etiología del transeuntismo es, por lo tanto, compleja y se debe a múltiples factores, pero los individuos con una socialización deficitaria y escasos recursos personales y sociales son los más vulnerables, pudiendo añadir a todo esto características específicas de su personalidad como reserva y aislamiento, dependencia de la aprobación del grupo y baja inteligencia. Asímismo hay que tener en cuenta la importancia del conocimiento de las variables predictoras del transeúntismo, ya que puede resultar muy importante para la prevención de situaciones de marginación y, cuando éstas ya se han producido, pueden ser útiles para la elaboración de Programas de intervención, ya que estas situaciones no tienen por qué ser irreversibles, pudiendo ser modificadas mediante una intervención adecuada sobre estos individuos.

\section{REFERENCIAS BIBLIOGRÁFICAS}

American Psychiatric Association (1995). DSM-IV. Manual diagnóstico y estadístico de los trastornos mentales. Barcelona: Masson.

Ayuntamiento de Madrid (1986). Transeúntes $e$ indigentes. Estados de necesidad y respuesta social. Concejalía de Servicios Sociales.

Caballo, V.E. (1987). Teoría, evaluación y entrenamiento de las habilidades sociales. Valencia: Promolibro.

Cassel, J. (1974). Psychosocial Processand Stress: Theoretical Formulations. International Journal of Health Services, 4, 471-482. 
Cobb, S. (1976). Social Support as a moderator of life stress. Psychosomatic medicine, 38, 300-310.

Díez Tomé, V., y Vielva Martínez, C. (1990). Transeúntes marginados en Cantabria. Necesidades y recursos. Dirección general de bienestar social. Diputación regional de Cantabria.

García Roca, J., Ayerbe, J., Perelló, F., Martínez, A., Belda, J.F., García Bacete, F., Musitu, G., y García Alverola, M. (1991). Pedagogía de la marginación. Madrid: Editorial popular.

González de Rivera, J.L. (1979). El estrés psicosocial como factor predisponente de enfermedad. En M. Ruiz, Psiquiatría preventiva. Barcelona: Ceppy.

Jiménez Tallón, M.A. (1987). La relación familiar como factor socioambiental significativo en el rendimiento escolar de una muestra de niños institucionalizados. Anales de Psicología, 5-17.

Jiménez Tallón, M.A. (1988). Estudio del estrés psicosocial en una muestra de niños institucionalizados. Anales de Psiquiatría, 193-197.
Kelly, J.A. (1992). Entrenamiento de las habilidades sociales. Bilbao: DDB.

Madrigal, P., Jiménez Tallón, M.A., y Madrigal, A. (1996). Programa de Intervención psicosocial sobre Transeuntes marginados en el Centro "Jesús Abandonado" de Murcia. IV Jornadas de Intervención Social. Instituto Nacional de Servicios Sociales, p. 639-649.

Mothner, I., y Weitz, A. (1989). Cómo abandonar las drogas. Barcelona: Martínez Roca.

Porot, M. (1954). L'enfant et las relations familiales. París: Paideia.

Rosenberg, M. (1979). Conceiving the self. New York: Basic Books.

Vielva Martínez, C. (1989). Transeúntes Marginados. Aspectos psicológicos. II Jornadas de Psicología de la Intervención social. Madrid.

Vielva Martínez, C. (1992). Estrés psicosocial $\mathrm{y}$ alteraciones emocionales en transeúntes marginados. Papel modulador de las redes de apoyo social. Intervención psicosocial, 1 , 79-86. 